\title{
Diagnóstico de establecimientos tradicionales del centro histórico de Quito- Ecuador, conforme a la normativa de zona especial turística
}

\section{Diagnosis of traditional establisments of the historic center of Quito-Ecuador, in accordance with the special tourist zone regulations}

María Dolores Quintana Lombeida

Universidad Tecnológica Equinoccial, Ecuador

Diego Alfredo Salazar Duque

Universidad Tecnológica Equinoccial, Ecuador

Autor por correspondencia: maria.quintana@ute.edu.ec, diegoa.salazar@ute.edu.ec

Fecha de recepción: 27 de febrero de 2018 - Fecha de aceptación: 15 de agosto de 2018

\section{Resumen \\ La presente investigación revela inicialmente un diagnóstico efectuado en la Zona Especial Turística del Centro Histórico de Quito, en las actividades turísticas de cafeterías y fuentes de soda, se aplicó dos cuestionarios. En el primero, se valoró los elementos para el cumplimiento de la Norma Técnica de Zona Especial Turística, y en el segundo, se evaluó los criterios de gestión, imagen comercial, producción y servicio de alimentos. El resultado evidenció el incumplimiento y las limitaciones en la prestación del servicio, lo cual reduce el nivel de satisfacción de los clientes y afecta la imagen de la Zona. Se constató las dificultades de ciertas infraestructuras patrimoniales en el cumplimiento de la Norma.}

Palabras Claves: zona especial turística; centro histórico de quito; producción; servicio; oferta gastronómica

\begin{abstract}
The present investigation initially reveals a diagnosis carried out in the Special Tourist Zone of the Historic Center of Quito, in the tourist activities of cafeterias and soda fountains, two questionnaires were applied. In the first, the elements for compliance with the Technical Standard of the Special Tourism Zone were evaluated, and in the second, the management criteria, commercial image, production and food service were evaluated. The result evidenced the breach and limitations in the provision of the service, which reduces the level of customer satisfaction and affects the image of the Area. The difficulties of certain heritage infrastructures in complying with the Standard were verified.
\end{abstract}

Key words: special tourism zone; centro histórico de quito; production; service; gastronomic offer 


\section{Introducción}

En 1978 la UNESCO declaró a Quito como patrimonio de la humanidad resaltando la conservación de la riqueza arquitectónica y artística de la ciudad. La Ley de turismo articulo 3 literal b, define como principios de la actividad turística la participación de los gobiernos provincial y cantonal para impulsar y apoyar el desarrollo turístico, dentro del marco de la descentralización.

La presente investigación analiza el actual comportamiento gastronómico en el Centro Histórico de Quito, considerado como una de las dos Zonas Especiales Turísticas que deben regirse a las normativas y reglamentos técnicos que requieren entidades de supervisión, entre ellas: ARCSA que exige el cumplimiento de los requisitos para su funcionamiento, establecidos en la normativa vigente. Y las reglas técnicas para la Zona Especial Turística Centro Histórico.

La ciudad de Quito tiene una afluencia turística aproximada de 680.000 visitantes (Municipio de Quito, 2014), un gran porcentaje de ellos frecuentan este lugar y acceden a los negocios que poseen infraestructuras patrimoniales que, lamentablemente, no cumplen con las diversas reglas que exige esta resolución, y mucho menos ciertas demandas culinarias que exige el mercado; por el contrario, existe una baja calidad en el servicio con una oferta culinaria muy limitada, falta de accesibilidad a servicios higiénicos, falta de calidad en el servicio de alimentos, administración empírica en la mayoría de pequeñas y medianas empresas. Como consecuencia de estos aspectos, la imagen del turismo gastronómico del sector aparece débil.

Para dar solución a esta problemática, se toma como base tres posturas importantes que se deben considerar en la gestión administrativa: la administración por objetivos promovida por Ducker (1954), la gestión estratégica propuesta por Ansoff y Brandenburg (1967), y la gestión por competencias postulada por David McClelland (1961). El primer aporte plantea la gestión de los procesos de administración por objetivos, los cuales deben ser planificados, controlados, apoyados permanentemente por la sinergia de esfuerzos enunciados y definidos por los resultados que se espera; la segunda, planteada por las decisiones organizacionales, enfocadas en las estrategias, las políticas, programas y procedimientos operativos; y finalmente, el tercer aspecto, el cual parte del análisis que se genera por la motivación humana, que según Charles Amstrong (1985), un modelo de gestión que se basa en competencias ${ }^{1}$ tiene que elevar la conductividad de la organización para mejorar su capacidad de enlazarse con el mundo exterior y los clientes, a través del lugar, el tiempo y el ambiente apropiado, que promueva un trabajo reflexivo y la efectividad de las interacciones.

En el estudio del turismo está inmerso un amplio glosario por la falta de propuestas universales de conceptualizaciones propias de la disciplina, por lo tanto en el alcance de los problemas que se presentan se postulan diferentes propuestas terminológicas según el especialista que la aborde (Boullón, 2006).

Para el estudio se consideró el enfoque especializado en la industria turística. Según González (2002), esta posición establece que las premisas que conforman un producto o servicio

\footnotetext{
${ }^{1}$ Competencias: hace referencia a las características de personalidad, devenidas en comportamientos, que generan un desempeño exitoso en puestos de trabajo (Alles, 2007)
} 
de calidad son cuatro; el primero, se refiere a los atractivos turísticos; el segundo, a la infraestructura adecuada para ofrecer el atractivo turístico (accesos); el tercero, a la estructuración de los establecimientos turísticos con recursos, productos y servicios idóneos (transportación, alojamiento o alimentación); y el cuarto, a la regulación y control de todo el proceso (entidad privada o pública).

Por otra parte, el diagnóstico de los establecimientos se validó a partir de Eiglier y Langeard (1993) con el planteamiento de la Servucción, que es la organización sistemática y coherente de todos los elementos físicos y humanos de la relación cliente empresa, necesaria para la realización de una prestación de servicio, cuyas características comerciales y niveles de calidad han sido determinados. Los elementos que conforman la Servucción son el soporte físico, el personal de contacto, el usuario, el servicio, la organización y los otros clientes. Alles (2005).

Finalmente, es indispensable la base del proyecto que se concretó a partir de la Ordenanza Metropolitana No 236, vigente desde el 15 de enero de 2009, la cual determina que la Empresa Metropolitana Quito Turismo, en coordinación con la Dirección Metropolitana de Planificación Territorial y Servicios Públicos, delimitará los espacios urbanos para el funcionamiento de establecimientos calificados como turísticos, que son alojamiento, expendio de alimentos y bebidas y otras vinculadas con el turismo.

En la misma ordenanza 236, en el capítulo IV, se menciona la creación de Zonas Especiales Turísticas (ZET), como territorios que reúnen "características y condiciones para la ejecución de una política turística común" donde el turismo sostenible, la mejora de la calidad de vida y la satisfacción de la demanda turística delimitan a La Mariscal y al Centro Histórico como las primeras ZET. Y el cumplimiento de la Resolución A012 que menciona los requisitos para los establecimientos ubicados en este territorio.

La presente investigación se basa en el desglose de la regulación y el control del proceso que se lleva a cabo en once establecimientos tradicionales del Centro Histórico de Quito.

Este instrumento se diseña de la siguiente manera: inicialmente se concreta el marco teórico en el que se refiere al Centro Histórico de Quito como Zona Especial Turística y las actividades de producción y servicio de alimentos en forma generalizada; en el segundo escenario se da a conocer la metodología empleada para el diagnóstico de los establecimientos y se desarrollan los resultados hallados; en el tercer momento se concretan las conclusiones y las nuevas perspectivas de investigación relacionadas con la plataforma temática. Para finalizar, se expone la bibliografía utilizada en el documento.

\section{Marco teórico}

\section{La Zona Especial Turística del Centro Histórico de Quito}

El Distrito Metropolitano de Quito está conformado por siete administraciones zonales: Calderón, Eloy Alfaro, Eugenio Espejo, Los Chillos, La Delicia, Manuela Sáenz, Quitumbe y Tumbaco; adicionalmente cuenta con dos zonas Especiales Turísticas (ZET), la primera el Centro Histórico y la segunda La Mariscal(Distrito Metropolitano de Quito, 2016). Las zonas 
especiales turísticas contribuyen a la promoción y desarrollo de la actividad turística, definen normas que buscan regular, fomentar y mejorar la calidad y los servicios.

El Municipio de Quito en la Resolución No A012 del 24 de octubre del 2014, señala que se ejecuten planes de gestión para las zonas especiales turísticas de La Mariscal y Centro Histórico de Quito, declarados como tales en la Ordenanza Metropolitana N. 236. A partir de la vigencia de la resolución, se definen plazos de cumplimiento para que posean dichas características los establecimientos involucrados:

$\checkmark$ Hoteles de lujo, primera y segunda categoría;

$\checkmark$ Hostales de primera y segunda categoría;

$\checkmark$ Pensiones de primera y segunda categoría;

$\checkmark$ Restaurantes y cafeterías de lujo, primera y segunda categorías;

$\checkmark$ Bares y fuentes de soda de primeras y segunda categoría;

$\checkmark$ Discotecas de primera y segunda categorías;

$\checkmark$ Salas de recepciones y banquetes de primera y segunda categorías;

$\checkmark$ Agencia de viajes mayoristas, internacionales, operadores y duales;

$\checkmark$ Transporte turístico (oficinas administrativas).

El punto de partida para efectuar el análisis de situación sobre el desarrollo turístico de la ZET Centro Histórico se sustenta en tres ámbitos que permiten abordar esta problemática: el espacio que es la base física y ambiental que aporta aptitud turística al territorio; el mercado con sus aspectos de oferta de producto, segmentos de consumidores y la competencia que brinda oportunidades para el desarrollo; y, las capacidades de gestión turística que hacen viable la estructuración de un destino con potencialidad y en condiciones de competitividad. (DMQ, 2014).

\section{La Resolución A012 destaca que:}

No se admitirá la implementación de bares y discotecas en la Zona Especial del Centro Histórico.

Los establecimientos que ofertan alimentos y bebidas deben practicar esta regulación para generar un buen servicio así como elevar o mantener su categoría entre establecimientos de primera y hasta segunda categoría eliminándose la opción de la tercera categoría y otras de menos representatividad.

La zona posee marcadas limitaciones en cuanto a equipamientos de apoyo en información, orientación, servicios higiénicos, y atención al visitante; 
Escasas facilidades turísticas con estándares de calidad que satisfagan las exigencias de una demanda de alto nivel de gasto; dificultades logísticas para el acceso y transporte de los visitantes, así como para el abastecimiento de los establecimientos, servicios básicos, de apoyo y operaciones turísticas; para mejorar el cumplimiento las Reglas Técnicas dividen al territorio del Centro Histórico en:

$\checkmark$ Sector A: Área turística cultural

$\checkmark$ Sector B: Área residencial-comercial

$\checkmark$ Sector C: Área residencial

El plazo de cumplimiento para la aplicación de estas sería el 31 de diciembre del 2015 , para la norma complementaria y en el caso del Sector A, territorio de la presente investigación, el plazo sería el 30 de abril del 2015.

La Regla Técnica define para los propietarios la obligación de su cumplimiento y desarrollo hasta alcanzar elementalmente la segunda categoría admisible para su normal funcionamiento.

\section{Cafeterías y fuentes de soda según la norma ZET}

En la Resolución A012 respecto de las cafeterías, se menciona que estas provean alimentos, bebidas frías y calientes, que es admisible el expendio de bebidas alcohólicas de moderación en cuanto acompañen otros alimentos. Mientras que respecto de las fuentes de soda describe que es admisible la venta de alimentos y bebidas fríos y calientes en el contexto de la comida rápida, no emite la necesidad de contar con servicio de comedor, las restricciones son el expendio de platos a la carta y venta de bebidas alcohólicas, en ambos casos, la calidad estaría determinada en función del tipo de los servicios.

La excepción que se contempla en todos los giros de negocio cita" Se exceptuarán ciertos parámetros técnicos relacionados con la infraestructura del bien en la aplicación de las nuevas Reglas Técnicas para Actividades Turísticas contenidas en los Planes de Gestión para las Zonas Especiales Turísticas de La Mariscal y Centro Histórico, en los establecimientos turísticos que funcionan en bienes inmuebles declarados como patrimoniales y/o inventariados, por lo que se tomará en cuenta dicha declaración y su carácter tradicional” (QuitoTurismo, 2014).

\section{El posicionamiento empresarial}

El posicionamiento en el mercado de un producto o servicio es la manera en la que los consumidores definen un producto a partir de sus atributos importantes, o sea, el lugar que ocupa el producto en la mente de los clientes en relación de los productos de la competencia. Si se tiene en cuenta las múltiples ofertas que existen y la información que estas emiten incitando a clientes reales y potenciales a comprar, se hace necesario para estos, algún tipo de organización para simplificar la decisión de compra. (Yinet Torres Peña, 2013 ). 
Los consumidores orientan su decisión de compra conforme su nivel de satisfacción en los bienes y servicios que adquieren, por su parte las empresas determinan las ventas y beneficios conforme ese interés del consumidor final, buscan estrategias que le otorguen valor a su oferta en el mercado en búsqueda del posicionamiento en el consumidor.

Los planes de marketing hacen hincapié en los aspectos psicológicos y sociales que condicionan al consumidor en sus compras, como el ahorro de tiempo, lo nutritivo, la salud, el aspecto físico, la conservación de la naturaleza o la seguridad, entre muchos otros. (Ismael Quintanilla Pardo, 2014).

La mejora de un producto o un servicio esta concatenado con la calidad que se le brinda a la satisfacción de la necesidad del cliente, en consideración de las capacidades que pueda otorgar el proveedor.

Para llegar a la mente del consumidor, es necesario identificar cuáles son las necesidades del mercado, lograr atraer los sentidos, enfocar tres elementos clave de éxito: la calidad de producto, la orientación del servicio y la imagen comercial frente a la múltiple competencia, a fin de incentivar la intención de compra en el potencial cliente (Urbano Medina Hernández, 2010).

Según el análisis del comportamiento del consumidor de Dubois y Rovira (1998), las estrategias genéricas de posicionamiento están dadas de tres maneras: el posicionamiento centrado en el consumidor, el posicionamiento basado en la competencia y el posicionamiento social del proyecto, de esta manera la oferta de productos y servicios aparece diversa y enfocada en la necesidad del cliente, según Dubois y Rovira (1998) para entender el comportamiento del consumidor es indispensable plantearse los cuestionamientos descritos en la siguiente tabla:

Tabla 1. Interrogantes para el análisis del comportamiento del consumidor

\begin{tabular}{ll}
\hline $\begin{array}{l}\text { ¿Por qué compra? } \\
\text { Razones de su comportamiento }\end{array}$ & Permite diseñar el producto de acuerdo a sus necesidades. \\
\hline $\begin{array}{l}\text { ¿Dónde compra? } \\
\text { Lugar donde efectúa la compra }\end{array}$ & Permite elegir a los distribuidores adecuados. \\
$\begin{array}{l}\text { ¿Cuándo Compra? } \\
\text { Período en que realiza la compra }\end{array}$ & Permite ofrecer oportunamente al consumidor el producto. \\
\hline $\begin{array}{l}\text { ¿Cómo compra? } \\
\text { Por precio, cantidad, otras condiciones }\end{array}$ & \\
\hline ¿Quién compra? & Permite determinar la forma de venta. \\
La persona que realmente compra & \\
\hline
\end{tabular}

Dubois B. y Rovira, A. (1998): comportamiento del consumidor. Comprendiendo al consumidor Pearson Prentice Hall, Madrid, $2^{a}$ edición.

En la Industria de alimentos y bebidas, el posicionamiento se enmarca en la higiene y manipulación del producto, en el servicio para su expendio y en las estrategias de 
comercialización. Particularmente en la ZET del Centro Histórico se accede a un mercado nacional y extranjero, proveniente principalmente de Colombia y Estados Unidos, por lo cual, la comprensión de los requerimientos del usuario es menester para crear lealtad e imagen positiva del destino.

\section{Higiene y manipulación del producto}

La percepción del consumidor frente al concepto de negocio de alimentos y bebidas es integral, por lo tanto, el cumplimiento de la premisa de higiene y manipulación del producto brinda al consumidor una garantía en su compra. Según la FAO (Organización de las Naciones Unidas para la Agricultura y la alimentación) y Codex Alimentario (1962), la responsabilidad de la seguridad alimentaria es de las entidades públicas y privadas y de todos los actores que intervienen en la transformación y comercialización de materia prima para el consumidor. Conforman así una cadena alimentaria logrando procesos responsables, identificando y controlando cada actividad inmersa en el producto.

Las Buenas Prácticas de Manufactura se implementaron en Estados Unidos en 1969, posteriormente se difundieron en el mundo con el fin de proveer a los usuarios una garantía en la adquisición de alimentos, hoy en día representan, en concordancia con los POES (Procesos Operativos Estándar de Saneamiento), un mecanismo indispensable para el aseguramiento de la calidad. Por lo tanto, los establecimientos de alimentos y bebidas de la Zona Especial Turística del Centro Histórico deberán ser intervenidos bajo esta norma, valorando las instalaciones, los equipos y utensilios, al personal y a las materias primas e insumos.

\section{El servicio}

La calidad del servicio se expresa en los esfuerzos permanentes que realiza una organización por satisfacer al cliente, convirtiéndose en una estrategia de diferenciación frente a la competencia.

Para Albretch (1992) representa los ciclos de servicio, fundamentados en los momentos de verdad. Este planteamiento se enfoca más como una ayuda para el productor de servicios. Juran (1990) y Deming (1989), identifican a la calidad como característica medible frente al cumplimiento de las necesidades del cliente.

Para las organizaciones prestadoras de servicio, la clave es tener una comprensión clara de los beneficios específicos que proporciona un servicio a los usuarios, identificar el objetivo del servicio y examinar cómo se modifica o se cambia medianamente un proceso de servicio específico; esto permite desarrollar una mejor comprensión del servicio fundamental y de los servicios primordiales que se ofrecen a los clientes. Lovelock (1997).

La fidelización y calidad en la atención a clientes y usuarios es una mezcla de cinco factores para el cumplimiento del éxito, si en algún caso la calidad del servicio no es oportuna el usuario lo detectará.

\section{Los cinco factores clave de la atención de cliente y usuario}




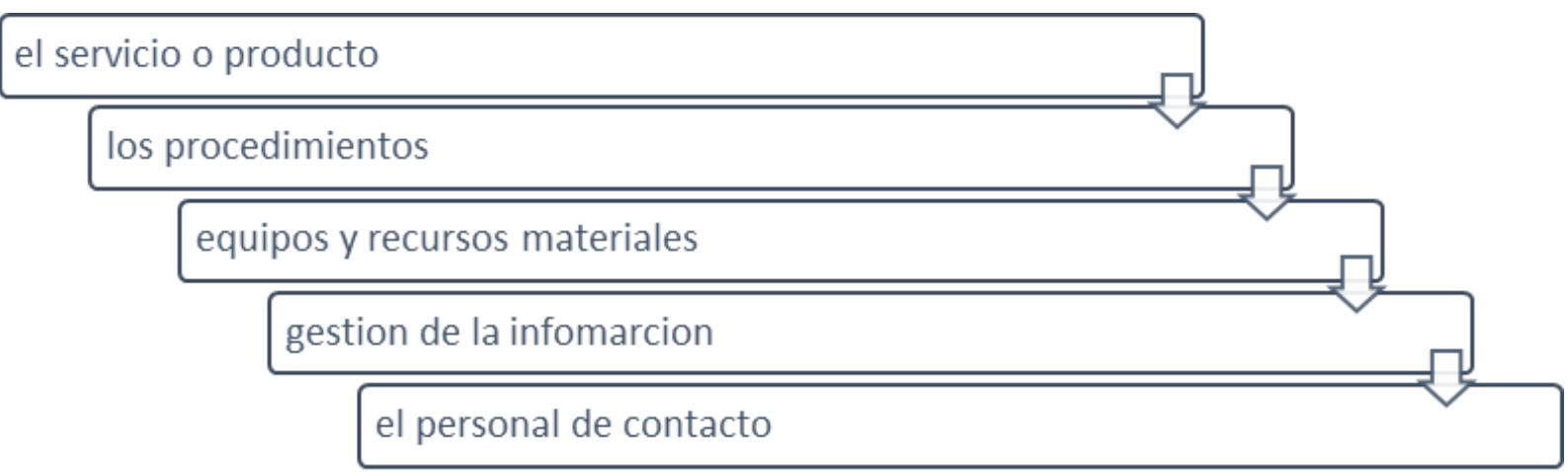

Fuente: Villa, Juan Pablo (2014). Manual de atención a clientes y usuarios. Editorial Profit

Todas las organizaciones están más que nunca obligadas a imprimir cambios en su cultura. Potenciar dentro de la compañía una cultura orientada al verdadero servicio al cliente.

Según (Villa, 2014) La comunicación asertiva es vital para la atención al cliente, la manera de manejar las quejas y reclamos identifica la solución a problemáticas que se presentan ante un cliente. Para ello según la Figura 1: la atención personalizada es clave, la cual debe ser integrada por: la bienvenida al cliente de manera acogedora, el intercambio de información de los servicios y productos de que brinda la empresa frente a las necesidades del usuario y la resolución de su necesidad concreta, la solución que se le otorga al cliente como y antes de terminar el contacto se debe asegurar la fidelización del cliente con la organización

\section{Fases de atención al cliente}

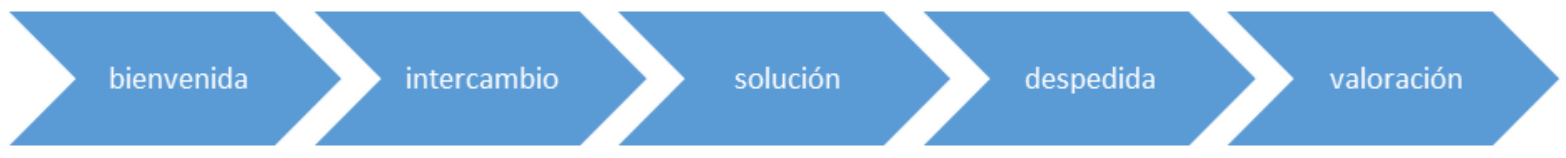

Elaborado por: autores

Fuente: Villa, Juan Pablo (2014). Manual de atención a clientes y usuarios. Editorial Profit

Un estudio reciente de la firma consultora estadounidense Booz Allen \& Hamilton, halló que el cliente generalmente decide cambiar de proveedor cuando identifica conflictos en el servicio, y no por presiones de precio o calidad del producto, lo cual se traduce en $40 \%$ de deserciones de clientes.

Por lo tanto, es indispensable para los establecimientos tradicionales del Centro Histórico realizar acciones que mejoren la calidad del servicio, frente a la competencia y al fortalecimiento del territorio.

\section{Estrategias de comercialización}

Para (Kotler, 2011), ganar a través del servicio superior implica la suma de varios atributos: rapidez, cordialidad, conocimiento, capacidad de resolución de problemas, y así 
sucesivamente. Cada usuario otorga diferente importancia al servicio que recibe. Las buenas empresas de servicios concentran su atención tanto en los clientes como en los empleados. La estrategia de marketing de servicios involucra servicio externo y marketing interno de motivación e interactividad con los empleados. El éxito está concebido por la creación de una ventaja competitiva diferenciadora que oferte calidad de servicio, y hallar mecanismos para elevar la productividad en el servicio (Philip Kotler, 2003).

El servicio es un bien intangible, en el que interactúan el personal en contacto con el cliente y el soporte físico, el cual plantea un sistema con todo el rigor necesario desde la concepción hasta la puesta en funcionamiento, para que el servicio sea de calidad. Según el modelo de Servucción de Eigler y Langeard (1993), el cliente como consumidor de servicio se integra al soporte físico, organización interna, personal y demás clientes. Por lo tanto, la percepción en el Centro Histórico de Quito es capaz de visibilizar los elementos que la organización ejecuta para su satisfacción y posterior lealtad.

\section{Objetivo}

- Evaluar las condiciones de producción y servicio de establecimientos de alimentos y bebidas, en contraste con las reglas técnicas de la Zona Especial Turística del Centro Histórico de Quito.

\section{Metodología}

La investigación es de carácter descriptivo exploratorio, compuesta de tres fases: diagnostico, evaluación y diseño. Para el desarrollo de la primera fase se utilizó dos cuestionarios, que incluyeron parámetros con criterios cuantitativos y cualitativos, los que se aplicaron durante el mes de agosto de 2015, a cargo de 22 estudiantes y 11 docentes de la Universidad Tecnológica Equinoccial.

El primer instrumento de recolección de información estaba conformado de 132 puntos categorizados en 5 temas:

$\checkmark$ Generalidades del negocio,

$\checkmark$ Características del propietario o administrador,

$\checkmark$ Gestión de los elementos administrativos legales y comerciales,

$\checkmark$ Diagnóstico de la infraestructura del local y

$\checkmark$ Procedimientos de buenas prácticas en higiene y manipulación de alimentos y bebidas,

Donde se consideró para su planteamiento a diferentes disciplinas Requisitos de Competencia Laboral. 2436-2008 Norma INEN, Buenas Prácticas de Manufactura, Principio administrativo: Planificación, Organización, Dirección y Control y Codex Alimentario. 
Conforme el cual con el $61 \%$ ya era considerado un establecimiento Satisfactorio en su desempeño.

El segundo cuestionario de evaluación desarrollado a partir de las Reglas Técnicas de Zona Especial Turística contaba con 97 ítems que incluían los siguientes tópicos: Identificación del establecimiento, Infraestructura, equipamiento, señalización, servicios, personal, mantenimiento, administración, legal, facilidades para personas con discapacidad, espacio público. Se utilizaron preguntas cerradas, y en ciertos casos, preguntas de carácter cuantitativo con escalas nominales continuas, valorándolos conforme el $70 \%$ como indicador de eficiencia.

\section{Desarrollo}

Para el desarrollo del proceso, Quito Turismo (entidad de control para el Distrito Metropolitano de Quito) identificó un universo de veinte giros de negocio que oferten alimentos y bebidas, donde su común denominador fue ser establecimientos tradicionales, y disponibilidad para brindar información de cada entidad; de este universo se manejó una muestra por conveniencia de 11 establecimientos.

Para validar dicha investigación se desarrolló dos encuestas piloto, que fueron aplicadas por tres especialistas en el área gastronómica e ingeniería de alimentos. Dichos documentos fueron planteados exclusivamente desde la Normativa ZET, considerando como base la categoría de los establecimientos evaluados, donde: seis de ellos fueron cafeterías, dos fuentes de soda, los tres establecimientos restantes (venta de bebidas típicas) correspondieron a establecimientos tradicionales del Centro Histórico de Quito.

El primero era un restaurante de segunda categoría, ubicado en el Sector de La Marín que no requería de cambio inmediato por ubicarse en la zona C; los otros dos establecimientos expedían bebidas preparadas en el Sector de San Francisco, por lo cual formaron parte del proyecto sin las exigencias de los ubicados en la Zona A.

El primer instrumento conforme a la normativa ZET se aplicó de acuerdo a la clasificación de los establecimientos designados, así:

Tabla 2. Instrumento de recolección de información a partir de la normativa ZET

\begin{tabular}{ll}
\hline Clasificación & Cafetería y fuentes de soda \\
\hline Categorías & Lujo, primera y segunda \\
Criterios & $\begin{array}{l}\text { Identificación del establecimiento, Infraestructura, equipamiento, señalización, servicios, personal, } \\
\text { mantenimiento, administración, legal, facilidades para personas con discapacidad, espacio público }\end{array}$
\end{tabular}

Ítems

97

Fuente: Los autores 
La segunda fase de la evaluación se valoró a partir de los datos obtenidos durante el diagnóstico del primer cuestionario, con los criterios estructurados por el investigador se llegaron a 132 ítems, donde se incluían interrogantes tales como capacidad máxima del establecimiento, número de empleados para el servicio, formación de los empleados en cada área de desempeño, disponibilidad de servicios higiénicos. Estos elementos fueron evaluados según la siguiente escala de evaluación:

\section{Tabla 3. Escala de evaluación}

\begin{tabular}{lc}
\hline ESCALA DE EVALUACIÓN & RAZÓN \\
\hline El establecimiento cumple con el estándar definido logrando alcanzar una evaluación de altamente & $81 \%$ a \\
satisfactorio, por lo tanto, puede funcionar y realizar las operaciones adecuadas tanto en producción & $100 \%$ \\
como en servicio, sin dejar de lado una planificación continua que permita mejorar sus procesos tanto & \\
administrativos como operativos. &
\end{tabular}

El establecimiento cumple con el estándar definido logrando alcanzar una evaluación de satisfactorio,

servicio, aun así deberá mejorar sus procesos con el fin de superar la calidad de la oferta, y generar planes de mejora con el propósito de que no decaiga en su evaluación y en los estándares que se requieren para brindar un producto y servicio de calidad.

El establecimiento cumple parcialmente con el estándar definido logrando alcanzar una evaluación de poco satisfactorio, por lo tanto, debe mejorar lo antes posible en toda su gestión, tanto administrativa como operativamente. Alcanzar una calificación de esta magnitud, conlleva a futuro comprometerse con la calidad del establecimiento, la satisfacción, la percepción y el valor del consumidor sobre la oferta que se brinda en el local. Esto puede generar una pérdida económica para el establecimiento.

$41 \%$ a $60,99 \%$

(nivel de riesgo)

$21 \%$ a $40,99 \%$ evidenciando faltas graves que comprometen seriamente la consecución de los objetivos empresariales. Alcanzar una calificación de esta magnitud, conlleva a futuro comprometerse con la calidad del establecimiento, la satisfacción, la percepción y el valor del consumidor sobre la oferta que se brinda en el local. Esto puede generar un cierre definitivo en las operaciones del establecimiento.

El establecimiento no cumple con el estándar definido logrando alcanzar una evaluación de ineficiente, por lo tanto, deberá realizar cambios en la totalidad de sus procesos tanto a nivel administrativo como operativo. Caso contrario el negocio no cumplirá con los requisitos para su funcionamiento.

Nota: Estos parámetros y escalas de evaluación son establecidos por Los autores, con el fin de evaluar y parametrizar el comportamiento de las empresas de alimentos y bebidas en la parroquia de Guayllabamba. También puede ser aplicado para evaluar cómo se encuentra el subsector

Fuente: Los autores

\section{Resultados}

Los resultados hallados conforme al primer cuestionario: 


\section{Indicadores cuestionario 1}

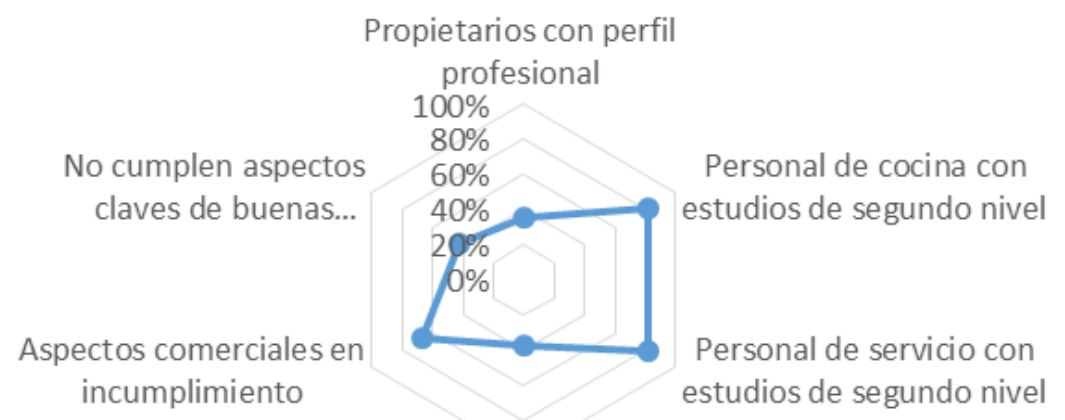

Indumentaria de personal

correcto

Figura 2. Resultados cuestionario 1

Fuente: Los autores

El empirismo prima en la Zona Especial del Centro Histórico de Quito, a nivel administrativo el ápice estratégico de los diferentes establecimientos está a cargo de personas sin estudios en el área. Por lo tanto el personal que trabaja en mandos medios tampoco recibe presión para su formación.

Al existir tanta afluencia turística al Centro Histórico de Quito, la calidad no ha mejorado, entidades de control municipales brindan capacitaciones gratuitas en competencias labolares pero no son de mayor interés de los proveedores porque; no les parecen atractivas las temáticas, los horarios no se ajustan a sus necesidades o la falta de utilidad de dichos procesos.

En cuanto al manejo higiénico de alimentos y menaje es riesgoso, en la mayoría de los casos no se utiliza uniforme completo y adecuado para la manipulación de productos, es prácticamente nulo el uso de mallas para el cabello y guantes descartables, las bebidas, frutas, vegetales y productos de bollería están expuestos al ambiente, el 50\% de establecimientos evaluados no posee servicios higiénicos lo cual no garantiza la elaboración higiénica de los productos ya que no existen espacios independientes para la higiene de las manos de alimentos y menaje.

En cuanto a la señalética no está controlada para el control de temperaturas de conservación y manipulación, esto se debe al escaso tamaño de los negocios ubicados en edificios patrimoniales que no permiten alteración en sus fachadas o ampliación de espacios. Dichos elementos argumentan la necesidad de exigir el cumplimiento de la Norma Técnica afín de exigir en áreas como manipulación de alimentos para garantizar la calidad de los productos.

Se concluye señalando la necesidad de una capacitación in situ, de mejoras en la línea de servicio, asesoría al cliente y de una herramienta que contribuya al mejoramiento de las 
actividades operativas y de servicio, por lo que hace falta material de apoyo en la manipulación de alimentos y señalética para cada establecimiento.

De esta manera se recomienda un documento como guía de bolsillo para el fácil manejo de los empleados, considerando gráficos y los procesos básicos de higiene y manipulación de alimentos enmarcados en tres puntos clave: el manipulador, los alimentos y las instalaciones.

Al respecto de las deficiencias halladas en la imagen comercial, se sugiere logotipos, tarjetas de presentación, manteletas, menús y propuestas de información para la publicidad de los diferentes giros.

\section{Resultados frente a la normativa ZET}

El común denominador que se identificó conforme al segundo cuestionario fue que el limitante en todos los negocios es que sean casas patrimoniales, lo que no permite la modificación en su infraestructura y accesibilidad para personas con capacidades especiales, viabilizar espacios para la adecuada manipulación de alimentos y comodidad del cliente, así como la incorporación de servicios higiénicos.

Otros aspectos que incumplen la Norma se refieren a la renovación de menús, ya que las cafeterías ofertaban platos a la carta lo cual no permite esta categoría; en cuanto a la infraestructura se detallaron elementos como energía, cableado y extracción.

La falta de identificación de placas con la tipología y categoría del establecimiento, así como señalética en torno a la restricción de consumo de tabaco no es visible en ninguno.

Solo dos establecimientos exhiben los precios de los productos los 9 restantes lo hacen a través de su carta.

En 6 establecimientos no se accede a servicios higiénicos, 2 de ellos cuentan con este servicio junto a las áreas de producción.

Los techos y pisos de 4 negocios cuenta con humedad y deterioro, la pintura no es lavable. El contacto con agentes contaminantes exteriores (polvo, smock) es visible en todos. reducido.

Ninguno cuenta con reservas de agua frente a la suspensión de este servicio por el espacio

Las sillas en 9 establecimientos no cuentan con separación de $50 \mathrm{~cm}$ como exige la norma.

Un solo negocio cuanta con dispositivo para la retención de aceite y grasa.

Solo 2 brindan mantenimiento a trampas de grasa, para evitar la contaminación de fuentes de agua y suelos. 
Ninguno cuenta con accesos externos e internos habilitados para personas con discapacidad motriz (rampas, barreras de seguridad y ascensor de ser necesario) por ser en su mayoría casas patrimoniales.

Se sugiere un plan de mejoras específico para cada local, con la finalidad de canalizar las necesidades inmediatas de avance, que permita superar la categoría que posee el negocio en la entidad reguladora Quito Turismo, hasta el momento de su clasificación, ya que no es admisible la tercera categoría.

El plan de mejoras deberá conformar los elementos de un plan de acción, donde se denoten las características generales del establecimiento, su categoría, las actividades urgentes a cumplir, los recursos necesarios para la aplicación, fechas límite de inicio y finalización de cada actividad, presupuesto, e indicador de seguimiento. Como se puede verificar en la tabla a continuación la mayoría de establecimiento presenta problemas de Infraestructura que es el causal para el incumplimiento de la norma ZET en varios aspectos.

\section{Tabla 4. Elementos comunes de mejora según normativa ZET}

\begin{tabular}{lc}
\hline Elementos & Criterio Norma \\
\hline Extracción de olores en baterías sanitarias & Infraestructura \\
Señalética para vías de evacuación & Infraestructura \\
Revisión de cableado eléctrico & Infraestructura
\end{tabular}

Sistema de extracción de olores y atrapa grasas

Revisión de sistema de iluminación natural y artificial

Superficies de acero inoxidable

Personal en higiene y uso de uniforme

Las sillas ocupadas entre mesas tienen una separación de $50 \mathrm{~cm}$.

Se deberá disponer de una mesa para personas con capacidades especiales, brindando todas las condiciones para este fin.

Los establecimientos de planta baja deben contar con rampas de accesibilidad.

Establecimientos con aforo de más de 90 personas, contar con baterías sanitarias específicas para personas con discapacidad
Infraestructura

Infraestructura

Equipamiento

Personal

Equipamiento

Infraestructura

Facilidades para personas con discapacidad

Facilidades para personas con discapacidad

Fuente: Los autores 


\section{Conclusiones}

Los establecimientos deben canalizar las necesidades a implementar hacer las mejoras pertinentes en las tres grandes premisas que fueron intervenidas. Se evidenció que los establecimientos involucrados alcanzaban en el análisis general un promedio del $45 \%$.

Se constató que los mayores conflictos en la aplicación de la Norma están dados por las limitaciones en la infraestructura, tal es el caso de las condiciones de arquitectura patrimonial que no cuenta con baterías sanitarias en su pequeñas instalaciones, el espacio dispuesto para la producción de alimentos permite, en ciertos casos, solo terminar los productos alimenticios, y deben contar con otro espacio para la pre elaboración.

En cuanto a la accesibilidad para brindar el servicio a personas con capacidades diferentes, se denotó que al respecto las habilidades, destrezas y conocimientos de los empleados y administradores deberían ser mayores y desarrollar procesos permanentes de capacitación en esta índole.

Es menester el interés por la capacitación permanente de los administradores y propietarios de carreras técnicas, que les permita contar con mayores estrategias de acción en sus establecimientos.

El elemento de gestión es excesivamente básico, los administradores deben cooperar en procesos de actualización que les faculte y permita obtener mayor viabilidad y rentabilidad en sus negocios, para su permanencia en el mercado como destino turístico preferencial para el turista nacional y extranjero.

\section{Bibliografía}

Agencia Nacional de Regulación, Control y Vigilancia Sanitaria. (s.f.). Emisión de permisos de funcionamiento. Recuperado el 09 de Junio de 2015, de http://www.controlsanitario.gob.ec/emision-de-permisos-de-funcionamiento/

Alcaldía del Municipio del Distrito Metropolitano de Quito. (2014). Plan de Gestión para la zona turística del Centro Histórico de Quito. Quito: Alcaldía del Municipio del Distrito Metropolitano de Quito.

Alles, M. (2007). Gestion por competencias: el diccionario. Colombia: Ediciones Granica S.A.

Boullón, R. (2006). Planificación del espacio turístico (4ta edición ed.). México: Editorial Trillas.

Chiavenato, I. (2002). Administración en los nuevos tiempos. Bogotá: Mc Graw Hill.

Distrito Metropolitano de Quito. (2015). Parroquia Guayllabamba. Obtenido de http://www.quito.com.ec/parroquias/index.php?option=com_content\&view=section\&id $12 \&$ Itemid $=19$ 
DMQ. (2014). Resolución A 012. Resolución A 012. Quito, Pichincha, Quito: DMQ.

El Telégrafo. (02 de julio de 2015). El sector servicios genera 1,9 millones de empleos. Recuperado el 29 de septiembre de 2015, de http://www.telegrafo.com.ec/economia/item/el-sector-servicios-genera-19-millones-de empleos.html

FAO/OMS. (2016). Codex alimentarios. Recuperado el 15 de enero de 2016, de http://www.fao.org/3/a-a0369s.pdf

INEN. (2008). Nte Inen-Iso 9001 Sistemas De Gestión De La Calidad. Requisitos. Quito. Obtenido de http://www.normalizacion.gob.ec/wp content/uploads/downloads/2014/NORMAS_2014/ACO/17122014/nte-inen-iso-9001 ext.pdf

INEN. (2013). Cpe Inen Codex 1 Principios Generales De Higiene De Los Alimentos. Obtenido de $\quad \mathrm{http} / / / \mathrm{www}$. normalizacion.gob.ec/wp content/uploads/downloads/2015/2015/DRO/16012015/cpe_inen_codex_cac_gl_69.pdf

Ismael Quintanilla Pardo, G. B. (2014). Comportamiento del consumidor. Barcelona: Editorial UOC.

Kotler, P. (2011). El marketiing según Kotler. Australia: Paidos.

López, N. (2003). Marketing en restauarción. Madrid: Editorial Sintesis.

Lovelock, C., \& Wirtz, J. (2009). Marketing de servicios: personal, tecnología y estrategia (6 ed. ed.). Naucalpan de Juárez: Pearson Educación.

Luthans, F. (1988). Successful vs. effective real managers. The Academy of management EXECUTIVE, 2(2), 127-132.

March, J., \& Simon, H. (1977). Teoría de la Organización . Barcelona: Editorial Ariel.

Merli, G. O. (2010). Escalas de medición en Estadística. Telos, vol. 12, núm. 2,, pág. 243-247.

Ministerio de Turismo. (2002). Ley de Turismo del Ecuador. Ecuador.

Municipio de Quito. (2014). Plan De Gestion Para La Zona Especial Turistica Del Centro Historico De Quito. Quito: Municipio de Quito.

Philip Kotler, G. a. (2003). Fundamnetos de marketing. México: Pearson.

QuitoTurismo. (2014). Reglas técnicas para cafeterías de la Zona Especial Turística del Centro Histórico. Quito. 
Taylor, F., \& Fayol, H. (1999). Principios de la administración científica. Administración industrial y general. Ateneo.

Urbano Medina Hernández, A. C. (2010). Cómo evaluar un proyecto empresarial: Una visión práctica. Madrid: Diaz de Santos.

Villa, J. P. (2014). Manual de atención a clientes y usuarios. Barcelona: Profit Editorial.

Yinet Torres Peña, G. G. (2013 ). Posicionamiento: Un lugar en la mente del consumidor de los servicios educativos. Editorial Académica Española. 